\title{
Acute Renal Infarction: An Underdiagnosed Disorder
}

\author{
Abdur Baig $^{\mathrm{a}}$, Elie Ciril ${ }^{\mathrm{b}}$, Gabriel Contreras ${ }^{\mathrm{a}}$, Oliver Lenz ${ }^{\mathrm{a}}$, Sonia Borra ${ }^{\mathrm{c}, \mathrm{d}}$
}

\begin{abstract}
Acute renal infarction is usually diagnosed when the triad of flank pain, hematuria and elevated lactate dehydrogenase is observed. Since the symptoms are non specific, diagnosis requires high degree of suspicion or may be missed or confused with renal colic. The incidence of ARI in emergency department visits is $0.007 \%$ whereas in autopsy series is reported $1.4 \%$ indicating the condition goes undiagnosed frequently. With the increase use of contrast enhanced computed tomography and magnetic resonance angiography the accuracy might be improving at the present time.
\end{abstract}

Keywords: Acute renal infarction; Flank pain; Hematuria; Lactate dehydrogenase; Contrast-enhanced computed tomography

\section{Introduction}

The incidence of acute renal infarction (ARI) in the emergency department (ED) visits has been reported as 7 in 100,000 [1]. It was found in $1.4 \%$ of cases in an autopsy study, whereas clinical diagnosis was made in $0.014 \%$ of those patients [2] suggesting that the condition is frequently missed and that a high degree of suspicion is needed to arrive at the diagnosis.

Manuscript accepted for publication February 13, 2012

${ }^{\mathrm{a}}$ Division of Nephrology and Hypertension, University of Miami, Miller School of Medicine, Miami, FL, USA

${ }^{\mathrm{b}}$ Melbourne Internal Medicine Associates, Melbourne, FL, USA

${ }^{\mathrm{c}}$ Department of Medicine, Kingsbrook Jewish Medical Center, Brooklyn, NY, USA

${ }^{\mathrm{d} C}$ Corresponding author: Sonia Borra, Department of Medicine, Kingsbrook Jewish Medical Center, 585 Schenectady Avenue, Brooklyn, NY 11203, USA. Email: sbnephro@gmail.com

doi: $10.4021 / \mathrm{jmc} 577 \mathrm{w}$
L Traube [3] published the first case of renal embolic disease in Germany in 1856 and since then multiple other case reports and case series have appeared in the medical literature.

The following case reports illustrate different risk factors that predispose to ARI. We also review the previously published case report series.

\section{Case Report}

\section{Case 1}

A 47-year-old man presented with right lower abdominal and back pain for three days associated with vomiting. The pain was throbbing in nature, had no radiation, and had an intensity of 10/10. He had smoked at least 5 cigarettes daily for the past 25 years, used heroin in the past, and currently received methadone $120 \mathrm{mg}$ daily. Physical examination was unremarkable except for severe right flank pain. His vital signs showed a blood pressure was $150 / 80 \mathrm{mmHg}$, pulse 60 beats per minute and regular, respirations 16 per minute, and temperature $36.5^{\circ} \mathrm{C}$.

Laboratory tests revealed hemoglobin of $14 \mathrm{~g} / \mathrm{dl}$, leukocyte count 7600 per $\mathrm{mm}^{3}$, neutrophils $80.5 \%$, platelets 241000 per $\mathrm{mm}^{3}$, serum creatinine $1.3 \mathrm{mg} / \mathrm{dl}$, aspartate aminotransferase 76 units/liter (normal 10 - 42), alanine aminotransferase was 65 units/liter (normal 10 - 40), lactate dehydrogenase (LD) 657 units/liter (normal 91 - 180). Urinalysis was strongly positive for blood, and urine protein was 300 $\mathrm{mg} / \mathrm{dl}$. Three sets of cardiac enzymes gave normal results. Total cholesterol was $196 \mathrm{mg} / \mathrm{dl}$ (normal 140 - 200), low density lipoprotein (LDL) $143 \mathrm{mg} / \mathrm{dl}$ (normal < 130), triglyceride $68 \mathrm{mg} / \mathrm{dl}$ (normal $35-160$ ) and homocysteine 6.9 micromoles per liter (normal $<11.4$ ).

The electrocardiogram showed sinus bradycardia at 52 beats per minute and inverted $\mathrm{T}$ waves in the inferior wall and chest leads. Echocardiogram revealed normal ejection fraction and no evidence of thrombus or vegetations. Impaired relaxation pattern of the left ventricular diastolic filling was observed.

Contrast-enhanced computed tomography (CECT) of 


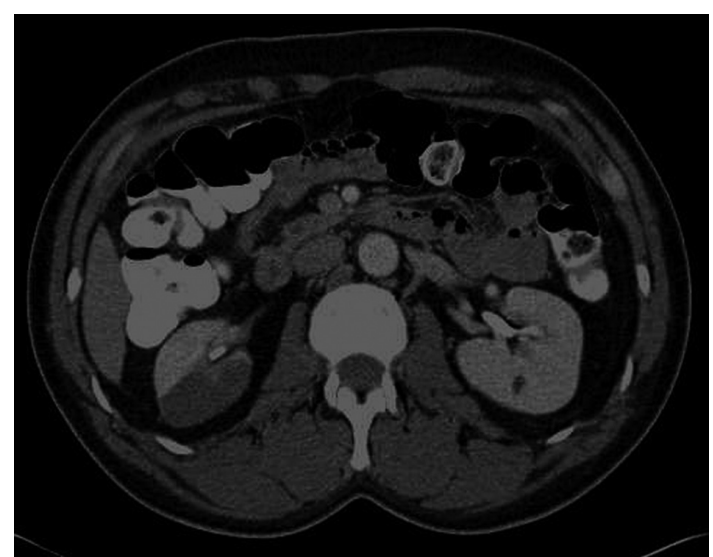

Figure 1. Contrast-enhanced computed tomography of the abdomen and pelvis showed right renal infarction.

the abdomen and pelvis showed right renal infarct with occlusion of the posterior branch of the right renal artery (Fig. 1). Gadolinium-enhanced magnetic resonance angiography (MRA) of the abdominal aorta and renal arteries confirmed right renal infarction involving the upper and posterior cortex.

He was anticoagulated with heparin and warfarin. His symptom improved significantly, and was discharged.

\section{Case 2}

An 89-year-old woman presented with right lower quadrant pain that started suddenly in the early morning hours, sharp in quality, 5/10 in severity, non radiating, and associated with nausea but no vomiting.

Her past medical history included atrial fibrillation, hypertension, congestive heart failure, and osteoarthritis. Medications on presentation were amlodipine, metolazone, enalapril, and aspirin. She had been taking warfarin in the past but had discontinued after an episode of toxicity. She never smoked or abused alcohol or illicit drugs. Her blood pressure was 160/60 $\mathrm{mmHg}$, pulse 67 beats per minute, irregular, respirations 16 per minute, and temperature $36.2^{\circ} \mathrm{C}$. She had right lower quadrant tenderness with no guarding or rebound. Bowel sounds were present.

Laboratory tests yielded a serum creatinine of $0.7 \mathrm{mg} / \mathrm{dl}$, hemoglobin13.1 g/dl, leukocyte count 6400 per $\mathrm{mm}^{3}$, platelets 213000 per liter, lactate dehydrogenase 1957 units per liter. Urinalysis was strongly positive for blood, and urine protein was $100 \mathrm{mg} / \mathrm{dl}$.

Electrocardiogram showed atrial fibrillation. Abdominal sonogram did not reveal abnormalities.

CECT scan of the abdomen and pelvis revealed poor enhancement in the lower two thirds of the right kidney and a focal filling defect in the distal right renal artery, suggestive of thromboembolism causing the infarct of the right lower two thirds of the kidney. Echocardiogram showed no evi- dence of thrombus or vegetations and the ejection fraction was $55 \%$. She was treated with heparin drip and later discharged on warfarin.

\section{Case 3}

A 41-year-old male was admitted to the hospital after suffering a gunshot wound, and had penetrating and blunt injuries. Past medical history and social history were unobtainable. On presentation blood pressure was $59 / 45 \mathrm{mmHg}$, pulse 98 beats per minute, respirations 40 per minute, and temperature $34.2^{\circ} \mathrm{C}$. He underwent splenectomy with repair of mesenteric injuries as well serosal tears of his colon.

Initial laboratory data showed a serum creatinine of 1 $\mathrm{mg} / \mathrm{dl}$, hemoglobin $12.4 \mathrm{~g} / \mathrm{dl}$, leukocyte count 7500 per mm $\mathrm{mm}^{3}$, platelets $153000 / \mathrm{L}$. No urinalysis was available. Computed tomography angiography (CTA) revealed hypoperfusion of the left kidney with multiple areas of wedge-shaped hypodensities representing possible infarcts. The left renal artery only demonstrated contrast enhancement on some of its branches suggesting renal artery injury.

Anticoagulation was initiated with heparin drip. Later he developed acute kidney injury and underwent multiple sessions of hemodialysis until renal function recovered. A follow-up CECT was done and revealed normal kidneys, at which time the heparin infusion was discontinued.

\section{Discussion}

The clinical presentation of ARI is nonspecific and may be confused with that of a renal colic. Patients commonly present to the ED with flank or abdominal pain associated with fever, nausea or vomiting, or may only present with acute onset of hypertension [4]. The prevalence of spontaneous kidney infarction in patients with new onset hypertension is $3 \%$ and the incidence is $0.18 \%$ per year [5]. The classic triad consists of persisting abdominal or flank pain; elevated serum LD and/or microscopic hematuria; and high risk of thromboembolism.

Laboratory studies reveal hematuria and proteinuria in most instances, and the blood levels of LD and the aminotransferases are frequently raised. Absence of hematuria indicates more serious loss of renal function [6]. Measurement of urinary LD may also be helpful.

The diagnosis is confirmed with CECT, angiography, or isotope scan. The sensitivity of computed tomography (CT) is $80 \%$, renal isotope $97 \%$ and angiography is $100 \%$ [6]. CT characteristically shows a perfusion defect with a mass effect representing the zone of infarct, sometimes surrounded by a cortical rim sign indicative of the zone of ischemia (functioning nephrons supplied blood via capsular collaterals). The isotope scan demonstrates absent or reduced blood flows to the affected area. Angiography can clearly delineate 
the lesion. Hilton S et al [7] correlated the CT findings with angiography and sonographic findings and concluded that the diagnosis can usually be made on the basis of specific CT findings. Angiography is valuable in helping to establish the cause and direct treatment. Sonography is less important in diagnosis but can be helpful in corroborating the CT findings. In case of high probability of renal infarction, Haung $\mathrm{CC}$ et al [8] suggested to obtain a CECT of the abdomen if no calculi are detected after unenhanced CT. They were able to make the correct diagnosis in $50 \%$ of cases during their first visit to the ED.

The pathogenesis of renal infarction is frequently due to thromboembolic events in the setting of dilated cardiomyopathy and atrial fibrillation. Frost L et al [9] followed 30,000 patients with atrial fibrillation over a 13 -year period and noted $2 \%$ incidence of renal artery embolism. Hazanov $\mathrm{N}$ et al [6] reported 44 cases of ARI in patients with atrial fibrillation over 18 -year period. Patients were mostly older than 60 years and had previous embolic events. Renal isotope scan was the most sensitive diagnostic test in this group of patients. Case 2 is an example of this complication although we could not detect any intracardiac thrombi on echocardiographic examination. Vegetations in infective endocarditis and cardiac tumors are other potential sources of emboli that could lead to a renal infarct, while case 1 illustrates an occlusion of the renal arteries due to thrombosis, which usually occurs with pre-existing renovascular disease.

Trauma or aortic interventions may also lead to ARI [10] as shown in case 3. Other risk factors include: clotting disorders, vessel anomalies such as fibromuscular dysplasia, kidney transplantation, antiphospholipid syndrome, Marfan or Ehlers-Danlos syndrome, autoimmune diseases including systemic lupus erythematosus or vasculitis, malignancies, and sickle cell disease. Cases of renal artery occlusion resulting from the use of cocaine [11] and infection with mucormycosis [12] have been described. It can be idiopathic. Bolderman $\mathrm{R}$ et al [13] identified sixteen patients (59\%) over 2.5 years who developed ARI without any structural or arrhythmic cardiac disease. Cigarette smoking increases the risk for arterial thrombosis. Nicotine causes vasoconstriction, decreases protein $\mathrm{C}$ activity and increases platelet aggregation and fibrinogen concentration. All of these effects may have contributed to the thrombosis of the abnormal renal artery in case 1 .

We reviewed ten published case series and tabulated the data in Table 1 (see supplement material, www.journalmc. org). There were a total of 213 patients. Patients most commonly presented with abdominal pain, followed by flank pain, nausea/vomiting, and fever. In all case series, laboratory findings were similar: hematuria, elevated LD, proteinuria and leucocytosis. The most common risk factor was atrial fibrillation, followed by history of thromboembolism, coagulation dysfunction and heart disease.

The treatment modalities include anticoagulation with heparin followed by warfarin, except when contraindications (such as aortic dissection) are present.

Thrombolysis and thrombectomy had been accomplished in patients with unilateral and bilateral occlusion but the results show only occasional improvement in renal function. In one case series, of seven patients who received thrombolysis, one developed end-stage renal disease, one had mild renal failure and two died [6]. In another case series, out of five patients, one patient's renal function did not recover or worsen during more than 1 year of follow-up [8]. In a case series reported by Korzets $Z$ et al [14], one patient had a combination of intravenous heparin and renal intra-arterial urokinase infusion, but no improvement was observed. The patient had a serum creatinine of 3.5 with a contralateral contracted kidney, required maintenance dialysis. Angioplasty and stent placement had been tried in some cases. The success of the intervention is limited by the duration of the ischemia.

The prognosis of ARI is determined by the etiology and the size of the infarct. In most series of unilateral disease there was not a significant loss of kidney function most likely due to compensatory hypertrophy of the remaining kidney tissue, but in most instances of bilateral infarct complete loss of renal function follows. Some patients develop persistent hypertension following ARI [5].

\section{Conclusion}

A high degree of suspicion remains the key for early diagnosis of ARI. Patients presenting with flank pain, hematuria and proteinuria as well as elevated level of LD should undergo CECT scan to obtain confirmation. Early anticoagulation may reduce symptoms and help preserve kidney function by preventing recurrence.

\section{Financial Support}

None.

\section{Conflict of Interest}

None.

\section{References}

1. Domanovits H, Paulis M, Nikfardjam M, Meron G, Kurkciyan I, Bankier AA, Laggner AN. Acute renal infarction. Clinical characteristics of 17 patients. Medicine (Baltimore). 1999;78(6):386-394.

2. Abreu S, Arruda H, Cury J. Concomitant renal and splenic infarction. Int Braz J Urol 2000;26:526-527. 
3. Traube L. Üeber den Zusammenhang von Herz- und Nierenkrankheiten. Berlin, Germany. A Hirschwald, $1856 ; 77$.

4. Fay MF, Nicholls MG, Richards AM. A man with severe, transient hypertension due to acute renal infarction. J Hum Hypertens. 1999;13(5):343-344.

5. Paris B, Bobrie G, Rossignol P, Le Coz S, Chedid A, Plouin PF. Blood pressure and renal outcomes in patients with kidney infarction and hypertension. J Hypertens. 2006;24(8):1649-1654.

6. Hazanov N, Somin M, Attali M, Beilinson N, Thaler M, Mouallem M, Maor Y, et al. Acute renal embolism. Forty-four cases of renal infarction in patients with atrial fibrillation. Medicine (Baltimore). 2004;83(5):292-299.

7. Hilton S, Bosniak MA, Raghavendra BN, Subramanyam BR, Rothberg M, Megibow AJ. CT findings in acute renal infarction. Urol Radiol. 1984;6(3-4):158-163.

8. Huang CC, Lo HC, Huang HH, Kao WF, Yen DH, Wang LM, Huang CI, et al. ED presentations of acute renal infarction. Am J Emerg Med. 2007;25(2):164-169.
9. Frost L, Engholm G, Johnsen S, Moller H, Henneberg EW, Husted S. Incident thromboembolism in the aorta and the renal, mesenteric, pelvic, and extremity arteries after discharge from the hospital with a diagnosis of atrial fibrillation. Arch Intern Med. 2001;161(2):272-276.

10. Tsai SH, Chu SJ, Chen SJ, Fan YM, Chang WC, Wu CP, Hsu CW. Acute renal infarction: a 10-year experience. Int J Clin Pract. 2007;61(1):62-67.

11. Bemanian S, Motallebi M, Nosrati SM. Cocaine-induced renal infarction: report of a case and review of the literature. BMC Nephrol. 2005;6:10.

12. Aarset H, Aasarod K, Bergan U, Angelsen A. Acute renal infarction in a woman with slight asthma. Nephrol Dial Transplant. 2001;16(8):1711-1712.

13. Bolderman R, Oyen R, Verrijcken A, Knockaert D, Vanderschueren S. Idiopathic renal infarction. Am J Med. 2006;119(4):356 e359-312.

14. Korzets Z, Plotkin E, Bernheim J, Zissin R. The clinical spectrum of acute renal infarction. Isr Med Assoc J. 2002;4(10):781-784. 